\title{
Light in darkness
}

\author{
We think dark matter exists because measurements of 'normal' matter would not otherwise make sense. \\ In this issue - our Insight on dark matter, offered jointly with Nature Physics - we showcase the various \\ techniques trying to make sense of it.
}

Without some kind of 'missing mass', galaxy rotation measurements would have galaxies spinning too fast to be stable; therefore something must be holding them together. This something could be an invisible form of matter, called dark matter. The clumpiness of large-scale galaxy structure would also be very difficult to explain without such dark matter, as would the measured anisotropy of the cosmic microwave background (CMB).

Of course, dark matter is not the only possible explanation. There are theories that do not invoke any invisible matter. Modified Newtonian dynamics (MOND), first proposed by Mordehai Milgrom, posits that Newton's laws do not apply to lowacceleration conditions, such as experienced by stars in the outer regions of galaxies. But despite some successes in explaining other phenomena, including the Tully-Fisher relation for a galaxy's total baryonic mass and its rotation velocity, there remains some missing mass in galaxy clusters within MOND models, which might well be dark matter.

So let us examine dark matter. In our Insight, we look at how the history of dark matter research goes hand in hand with the history of cosmology. From the 1930s, the evidence for dark matter increased to a point when, in the 1970s, the cosmology community had to take action. This ground is covered by Jaco de Swart, Gianfranco Bertone and Jeroen van Dongen in their Review Article (Nat. Astron. 1, 0059; 2017). In his Perspective, James Peebles recalls the convergence of evidence and ideas in the 1980s that made the theory of nonbaryonic dark matter compelling (Nat. Astron. 1, 0057; 2017). And let us not forget Vera Rubin - an obituary by Neta Bahcall was recently published in Nature (542, 32; 2017). Her galactic rotation curves in the optical range, measured with Kent Ford in the 1970s, helped to convince the community that something was amiss between the measured and predicted rotation rates.

On the modern experimental front, efforts have focused on sterile neutrinos, weakly interacting massive particles (WIMPs) and massive astrophysical compact halo objects (MACHOs; which could be brown dwarfs, black holes or other objects that emit little or no light). Axions are also a viable candidate. They are neutral, very weakly interacting particles that may explain why there is no charge-parity symmetry in the strong interaction. What is appealing about axions (and other axion-like particles) is the possibility for their detection in small-scale experiments. Mature axion dark matter searches — such as the Axion Dark Matter eXperiment, Any Light Particle Search and CERN Axion Solar Telescope, for example - have placed limits on axion-photon coupling. But as we cannot cover all dark matter candidates in one go, we focus on WIMP searches, which have made the most experimental progress.

WIMPs are a class of stable, neutral particles. The most popular are those from supersymmetry models that extend the standard model, such that each standard model particle has a supersymmetric partner. In one type of experiment, the idea is that if these particles exist, Galactic WIMPs must be passing through Earth, and would scatter off target nuclei, for instance within a noble-liquid target. Recoiling nuclei would emit light, which can be detected by scintillators. Jianglai Liu, Xun Chen and Xiangdong Ji (Nat. Phys. 13, 212-216; 2017) discuss the status of such direct detection experiments, using noble liquids and solid-state detectors. At higher energy scales, particle colliders are smashing particles together and looking for WIMPs in the annihilation or particle decay channels. In their Progress Article, Oliver Buchmueller, Caterina Doglioni and Lian-Tao Wang describe efforts at the Large Hadron Collider at CERN (Nat. Phys. 13, 217-223; 2017).

Indirect detection methods involving astrophysical sources look for imprints of dark matter on the energy spectra or spatial distribution of gamma-ray photons or charged cosmic rays. These particles come from the Galactic Centre, Milky Way halo, dwarf spheroidal galaxies, galaxy clusters and the extragalactic gamma-ray background (from pulsars, active galactic nuclei, star-forming galaxies and so forth). Jan Conrad and Olaf Reimer (Nat. Phys.
13, 224-231; 2017) bring us up to speed on the current status of indirect dark matter detection. Besides cosmic and gamma rays there are other messengers that could tell us something about dark matter: highenergy neutrinos. Neutrino telescopes such as IceCube can place limits on WIMP annihilation cross-sections, complementing those determined from direct and collider experiments. In his Review Article, Francis Halzen discusses the present and future of high-energy neutrino astronomy (Nat. Phys. 13, 232-238; 2017).

If dark matter holds visible matter in galaxies tighter together, there is another dark entity that does the opposite. We have known since the 1920s that the Universe is expanding at a rate determined by Hubble's constant, as explained by Barbara Ryden (Nat. Phys. 13, 314; 2017). But in the late 1990s, type Ia supernovae measurements revealed that the expansion rate is accelerating. Assuming the model of Big Bang cosmology is correct, the acceleration of the expansion of our (observationally) flat Universe implies that something must be doing the pushing - dark energy - or again we need to modify the theory of gravity. But let us hold onto Einstein's general relativity for now, and consider dark energy, as it is measurable, albeit indirectly. This force only interacts with gravity and is invisible to electromagnetic radiation; it pushes visible and dark matter alike. Precision measurements of galaxies and galaxy clusters reveal the underlying galaxy structure; the CMB - the geometry of the Universe; and thousands of supernovae the expansion rate of the Universe. The Dark Energy Survey maps these effects to determine the expansion history of the Universe, explains Joshua Frieman (Nat. Astron. 1, 0066; 2017). Meanwhile, Keith Vanderlinde writes about a radio interferometer, the Canadian Hydrogen Intensity Mapping Experiment, which is preparing to scan half the sky, and over 4 billion years of cosmic history, to probe the increasing expansion rate (Nat. Astron. 1, 0037; 2017).

The Universe is full of darkness and we are just beginning to make sense of it. 